\title{
iBeam: Intelligent Client-Side Multi-User Beamforming in Wireless Networks
}

\author{
Yongjiu $\mathrm{Du}^{1}$, Ehsan Aryafar ${ }^{2}$, Joseph Camp ${ }^{1}$, and Mung Chiang ${ }^{3}$ \\ ${ }^{1}$ EE Department, Southern Methodist University, Dallas, TX, USA \\ ${ }^{2}$ Intel Labs, Santa Clara, CA, USA $\quad{ }^{3}$ EE Department, Princeton University, Princeton, NJ, USA
}

\begin{abstract}
Frequently, client-side wireless devices have a view of multiple WiFi access points, whether from open residential and commercial networks, corporate networks, or mesh networks. Given the increasing number of radios and antennas in today's wireless devices, residual capacity from these multiple APs could be leveraged if client devices communicate with multiple APs simultaneously. In this paper, we exploit multi-user multi-input multi-output (MU-MIMO) technology to improve throughput and reliability in both directions of a wireless connection. For uplink, we use multi-user beamforming to enable the client devices to send multiple data streams to multiple APs simultaneously. For downlink, we leverage interference nulling technology to allow the client devices to decode parallel packets from multiple APs. This iBeam system requires no changes to existing APs or backhaul networks and is compatible with the IEEE 802.11 standards. We experimentally evaluate iBeam and show significant throughput improvements over both single-AP connections and multi-AP connections in a time division mode. The client's reliability and stability are also significantly improved due to the multi-AP diversity gain.
\end{abstract}

Index Terms-Multi-user; Multi-AP; Beamforming; Interference Nulling; Channel Reciprocity; WiFi Networks; Rate Adaptation;

\section{INTRODUCTION}

Wireless devices have recently offered an increasing number of radios and antennas, which have been leveraged in recent work and $\mathrm{WiFi}$ standardization efforts for improved performance from access points to clients [1], [2]. However, despite similar hardware trends for client-side devices, the multiple radios and antennas have yet to be fully leveraged to improve the uplink or downlink performance. In particular, the radio and antenna growth has enabled multi-input multi-output (MIMO) technologies to be leveraged from APs to clients, thereby improving capacity. Beamforming is one such MIMO technology which can improve the received signal strength of the intended user and reduce interference to the unintended users, as has been studied recently [3], [4]. Moreover, multiuser beamforming (MU-beamforming) can achieve additional throughput improvement by properly setting the steering matrix of the transmitting links to enable parallel data transmissions [1], [5]. Another key MIMO technology is interference nulling and cancellation, which allows a receiver to decode multiple parallel streams from different transmitters [6]-[8].

Other work has made use of users frequently being in range of multiple access points with a plethora of available networks in homes or businesses or larger corporate or mesh networks. In [9], for example, the authors introduced a protocol that enables the client device to aggregate the backhaul bandwidth of multiple APs that can be seen by the client, in a time division mode. ViFi, proposed in [10], opportunistically exploits the diversity of multiple APs to minimize disruptions and supports interactive applications for client devices. Given the increasing number of radios in today's wireless devices, residual capacity from these multiple APs could be further exploited if client devices communicate with multiple APs simultaneously as opposed to each AP being multiplexed over time. With the aforementioned MIMO technologies, these multi-AP approaches could be vastly improved to provide increased throughput and reliability. Namely, instead of communicating to multiple APs in a time division mode, with MIMO technology, the client device can maintain connections to multiple APs simultaneously. Adya et al. leverage the simultaneous use of multiple wireless network cards on nonoverlapping frequency channels to communicate with multiple APs, achieving significantly improved throughput and latency [11]. However, this scheme does not leverage the efficiency of MIMO and occupies multiple channels, resulting in a large spectral footprint.

Motivated by the high cost of spectrum in licensed bands and high usage in unlicensed bands, we propose iBeam, a wireless system that uses a single channel for multi-AP communication, which can be easily scaled by additional channel availability. In iBeam, the client device can transmit parallel data streams to and receive multiple data streams from multiple WiFi APs simultaneously. We take advantage of channel reciprocity and passive overhearing of control and broadcasting packets transmitted by APs, to avoid incurring channel-state-information (CSI) overhead by the client, thereby improving system efficiency. For MU-beamforming system, power allocation among all the selected beamformees is challenging, especially when time-varying channel and rate adaptation are considered. For the downlink, we use interference nulling technology to enable multiple APs transmitting multiple streams to the client simultaneously. We implement it on the WARP FPGA platform [12] and evaluate it over the air. We experimentally show that iBeam achieves significant improvement in terms of both throughput and reliability. With just 2 antennas on the client device, we achieve an average improvement of 50 percent for the uplink direction and 80 percent for the downlink direction. Also, with multiple-AP connections and smooth handoff, there is almost no outage for the connection.

While there is no shortage of works on advanced physical layer MIMO techniques (e.g., [1], [8]), our goal here is to design and experimentally evaluate a system which leverages such techniques from the point of view of the client to 
improve throughput, reliability, and stability. This is in contrast to and complementary with other works which improve the access point performance [6], [13]. In particular, the main contributions of our work are as follows:

- We propose iBeam, a system on client devices to communicate with multiple APs simultaneously, without changing the network infrastructure, access point protocols, or WiFi standard.

- We implement iBeam on a hardware platform by exploiting multi-user beamforming to enable the client device to send multiple uplink data streams to multiple APs simultaneously. For downlink, we use interference nulling technology to allow the client device to decode parallel packets from multiple APs.

- We experimentally evaluate this iBeam system and show significant throughput improvements over single-AP connections or multi-AP connections in a time division mode. The results show an average throughput improvement of 50 percent and 80 percent, for uplink and downlink, respectively.

- We also experimentally analyze the stability and reliability of the iBeam system. In particular, with mobile handoff, we show that iBeam is almost independent of client speed, whereas more traditional techniques suffer severe degradation and long periods of disconnection with user mobility.

The rest of this paper is organized as follows. Section II introduces the iBeam system. In Section III, we present the uplink framework for iBeam, including multi-user beamforming technology, and the method to select the optimal subset of APs for which the client device should connect. We describe the iBeam downlink framework in Section IV. Experimental settings and results are shown in Section V. Then, related work is provided in Section VI. Finally, we conclude in Section VII.

\section{IBEAm System Model}

In this section, we present the system model for iBeam, which enables a client device to maintain multiple WiFi access point connections on the same channel, as shown in Figure 1. The client device is equipped with multiple antennas, which are used to connect to multiple APs. In doing so, there are three distinct advantages over a traditional MIMO scheme: $(i)$ clients can remain connected in mobile scenarios via seamless handoffs, (ii) MIMO gains are greater with increased antenna separation on the senders and receivers (as opposed to both having co-located antennas), and (iii) backhaul bandwidth can be aggregated to achieve the capacity of the wireless network. In this WiFi network, the client device is assumed to be able to see several APs operating on the same channel.

For uplink, the client device first selects an optimal subset of APs and then transmits multiple data streams to the selected APs to improve the capacity. For downlink, the associated APs are potentially able to transmit packets simultaneously to the client device, if there are packets in the buffer of each AP. The number of APs that the client device can select does not exceed the number of antennas on the client device. Because of the connections to multiple access points, the handoff is smooth when the client device moves out of the coverage of an AP

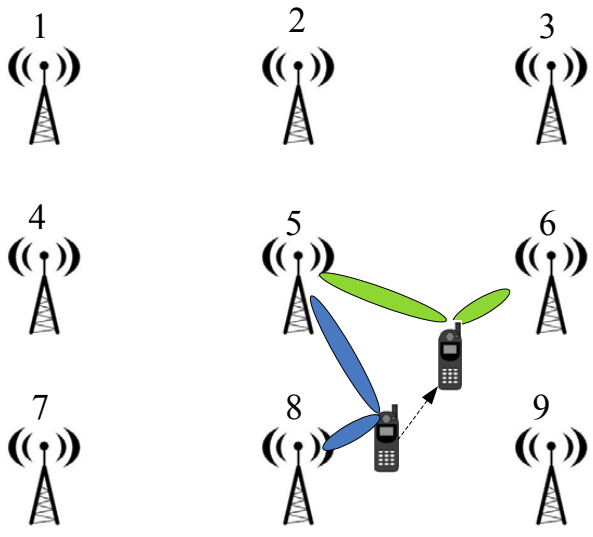

Fig. 1: A client device has connections to multiple APs.

and moves into the coverage of another AP. The client device can maintain at least one connection to an AP, while making handoff between other APs. Taking the case in Figure 1 as an example, the mobile client is initially connected to AP 5 and AP 8. When the client device moves towards AP 6, the channel between AP 8 and the client device deteriorates and the channel between AP 6 and the client device improves. The client devices then initiates a handoff from AP 8 to AP 6, while always maintaining the connection to AP 5.

In reality, the APs may be densely planned in a large population area, or sparse in remote areas. As a result, the APs may not be able to sense one another. According to carrier sense multiple access (CSMA), only one AP is able to win the time slot and transmit if the APs can sense each other. In the case that the APs cannot hear each other and the client device can see multiple APs, multiple APs can transmit to the client device simultaneously. The client device can decode the parallel signals from the APs, leveraging interference nulling technology.

For uplink, the client device uses MU-beamforming with a zero-forcing method to transmit multiple beams, with different packets on the beams. Zero-forcing eliminates the interference between the APs, which results in less complexity since the beamforming and power allocation algorithm are independent of one another. For iBeam, the client device can communicate to each AP with a separate beam. It is also possible for an iBeam client device to maintain a two-stream connection to one AP and one-stream connections to other APs. In other words, the number of streams between the two APs can be heterogeneous.

The iBeam system requires no additional coordination between the multiple APs in use at a given time (i.e., the multiAP intelligence comes from the client-side device) and needs no changes to each AP's protocols (e.g., message passing) or standards (e.g., iBeam is compatible with IEEE 802.11). The traffic management when using multiple APs is well discussed in prior works [9], [11], [14], and we can abstract the client device to appear as multiple virtual interfaces, for both the upper layer applications and the APs. We will then configure each virtual card to connect to a different AP. iBeam also requires the client device to encode/decode multiple spatial 
streams in parallel, which has been adopted in IEEE 802.11 $\mathrm{n} / \mathrm{ac}$ devices.

\section{IBEAM UPLINK}

For the uplink direction of the iBeam system, we apply multi-user beamforming on client devices to transmit parallel data streams to a set of available APs. To avoid injecting additional overhead, we use control and broadcasting packets from the AP (e.g., beacons) to measure the downlink channel and leverage channel reciprocity to estimate the uplink channel. In Section V, we experimentally evaluate the channel reciprocity assumption with respect to channel quality. According to the channel gains, the set of APs is chosen in such a way to obtain the optimal capacity. When the client device moves, the channel between the client device and the connected APs will be altered. The client device then needs to update the channel information and adaptively change the beamforming parameters to maintain the zero-forcing conditions. Furthermore, the selected optimal subset of connected APs may also change. As a result, the client device will initiate mobile handoffs between APs to maintain the optimal performance.

\section{A. Multi-User Beamforming}

In this section, we describe multi-user beamforming (MUBeamforming), which is a key uplink technology in our iBeam system. Assume one client device with $M$ antennas beamforms to $N$ APs, and each AP has one antenna. For MUBeamforming, we can express the received signal at $\mathrm{AP} i$ as:

$$
\begin{aligned}
y_{i} & =\boldsymbol{h}_{i} \times\left[\boldsymbol{q}_{1}, \boldsymbol{q}_{2}, \ldots, \boldsymbol{q}_{N}\right] \times \boldsymbol{x}+\boldsymbol{n} \\
& =\left(\boldsymbol{h}_{i} \boldsymbol{q}_{i}\right) x_{i}+\sum_{j \neq i} \boldsymbol{h}_{i} \boldsymbol{q}_{j} x_{j}+\boldsymbol{n} \\
& =\left(\boldsymbol{h}_{i} \boldsymbol{q}_{i}\right) \sqrt{P_{i}} s_{i}+\sum_{j \neq i} \boldsymbol{h}_{i} \boldsymbol{q}_{j} \sqrt{P_{j}} s_{j}+\boldsymbol{n}
\end{aligned}
$$

Here, $\boldsymbol{x}=\left[x_{1}, x_{2}, \ldots, x_{N}\right]^{T}$ is the transmit signal vector for all $N$ receivers. $\boldsymbol{h}_{i}=\left[h_{i, 1}, h_{i, 2}, \ldots, h_{i, M}\right]$ is the channel vector from the beamformer to beamformee $i . \boldsymbol{q}_{i}=$ $\left[q_{i, 1}, q_{i, 2}, \ldots, q_{i, M}\right]^{T}$ is a steering vector from the beamformer to beamformee $i . \boldsymbol{n}$ is a vector of additive noise and may include interference from other networks. $s_{i}$ is the normalized transmitted signal with transmit power $P_{i}$.

In Equation (1), the first and second terms represent the intended transmission and interference from other APs, respectively. Each receiver decodes the transmitted packet by treating both the interference and noise as an additive Gaussian noise. Then, the achieved sum rate is:

$$
R=\max _{\boldsymbol{q}_{n}, P_{n}} B \sum_{n=1}^{N} \log \left(1+\frac{P_{n}\left|\boldsymbol{h}_{n} \boldsymbol{q}_{n}\right|^{2}}{\sum_{j=1, j \neq n}^{N} P_{j}\left|\boldsymbol{h}_{n} \boldsymbol{q}_{j}\right|^{2}+\sigma^{2}}\right)
$$

subject to $\sum_{n=1}^{N}\left\|\boldsymbol{q}_{n}\right\|^{2} P_{n} \leq P$.

To determine the optimal $\boldsymbol{q}_{n}$ and $P_{n}$ to achieve the maximum rate is highly complex, especially when there is a large number of receivers. Zero-forcing is a sub-optimal but comparatively simpler solution for this problem [5], [15], [16]. The key idea is to properly select $\boldsymbol{q}_{j}$ to make $\boldsymbol{h}_{i} \boldsymbol{q}_{j}=0$ for all $j \neq i$.
Assume the client device transmits packets to a subset of $S$ APs out of $N$. Let $\boldsymbol{H}(\boldsymbol{S})$ and $\boldsymbol{Q}(\boldsymbol{S})$ denote the corresponding sub-matrices of $\boldsymbol{H}=\left[\boldsymbol{h}_{1}^{T} \cdots \boldsymbol{h}_{N}^{T}\right]^{T}$ and $\boldsymbol{Q}=\left[\boldsymbol{q}_{1} \cdots \boldsymbol{q}_{N}\right]$, respectively. The solution of $Q(S)$ to obtain zero-forcing is the pseudo inverse of $\boldsymbol{H}(\boldsymbol{S})$ :

$$
\boldsymbol{Q}(\boldsymbol{S})=\boldsymbol{H}(\boldsymbol{S})^{*}\left(\boldsymbol{H}(\boldsymbol{S}) \boldsymbol{H}(\boldsymbol{S})^{*}\right)^{-1}
$$

Then, we can choose $P_{j}$ based on the water-filling algorithm to maximize the total rate, or use other power allocation algorithms with consideration of fairness between the APs.

The client device is able to control the packet size for each AP to allow transmissions to different APs to end at the same time, which increases spatial reuse, and allows the ACKs mechanism to be simple. After successfully receiving the packets, the APs send ACKs back to the client device. The client device then decodes the ACKs simultaneously, leveraging the interference nulling technology described in Section IV.

\section{B. Semi-Orthogonal AP Selection}

As discussed above, we nullify interference from the transmissions to different APs by forcing $\boldsymbol{h}_{k} \boldsymbol{q}_{j}=0$, for $k \neq j$. In order to maximize the transmit power efficiency for AP $j$, a preferred steering vector should satisfy $\boldsymbol{q}_{j}=\boldsymbol{h}_{j}^{*} /\left|\boldsymbol{h}_{j}\right|$. Therefore, when $\boldsymbol{h}_{k} \boldsymbol{h}_{j}^{*}=0$, for $k \neq j$, both conditions are satisfied, and both the beamforming gain and the multi-user diversity gain can be obtained. This means that, for different APs, if their channel vectors to the client device are orthogonal to each other, we obtain the optimal gain. However, in practice, it is hard to find the channel vectors that are absolutely orthogonal to each other, especially for small number of APs. A sub-optimal but feasible solution is to find the subset of APs that have the best orthogonality, which forms a semiorthogonal subset of APs.

For ease of presentation, each client device is equipped with two antennas, which allows it to communicate with two APs simultaneously with MU-beamforming. Upon entering the network coverage area, the client device detects all the potential APs for connection and obtains the channel state information to each AP. Among the detected APs, it finds the two optimal APs by jointly considering the channel gains and the channel orthogonality. To do so, we propose (and will implement and evaluate) the following greedy algorithm:

1) For each AP seen by the client device, calculate the norm of the channel gain $\rho_{i}=\left\|\boldsymbol{h}_{i}\right\|$ and select the AP that maximizes $\rho_{i}$.

2) For each of the remaining APs, find the value of $\alpha_{i}=$ $\frac{\left|\boldsymbol{h}_{i} \boldsymbol{h}_{j}^{*}\right|}{\left\|\boldsymbol{h}_{i}\left|\left\|\mid \boldsymbol{h}_{j}\right\|\right.\right.}$ and choose the AP with the value of less than or equal to a constant $\alpha$. If none of the APs satisfy this condition, then the client device only communicates to the AP selected in step 1.

3) If at least one AP satisfies the requirement in step 2, we calculate $\eta_{j}=\boldsymbol{h}_{j}-\frac{\boldsymbol{h}_{j} \boldsymbol{h}_{i}^{*}}{\left\|\boldsymbol{h}_{i}\right\|^{2}} \boldsymbol{h}_{i}$ and choose the AP with the maximum $\left|\eta_{j}\right|$ as the secondary AP.

This method easily scales to more antennas and more simultaneous APs for even greater levels of performance. 


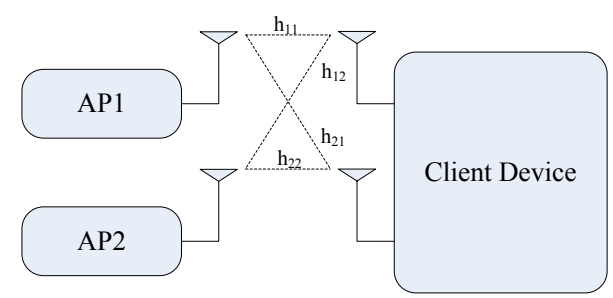

Fig. 2: Two APs transmit packets to the same client device simultaneously.

\section{AP Adaptation}

When the client device moves, the channel gains and orthogonality between the client device and the APs are altered. In order to maintain a zero-forcing transmission, the client device needs to adapt the beamforming parameters to the changing channel conditions. Moreover, the changing channel conditions may result in a different optimal subset of connected APs. The client device may reselect the optimal subset of APs according to the updated channel state information. If the selected subset of APs changes, the client device needs to initiate the handoff process between the disconnecting APs and the newly selected APs, to optimize the system performance. In order to maintain the service quality, there are generally two rules to follow. First, the client device needs to maintain at least one connection to an AP during the handoff between other APs to avoid service disruption. Second, the handoff should not be too sensitive to the channel quality, to avoid handing off too frequently. We can apply existing handoff methods as described in [17] to successfully adapt APs.

\section{IBEAM DOWNLINK}

A device with multiple antennas can potentially decode multiple parallel packets simultaneously on the same channel. With the knowledge of CSI, we can apply interference nulling with or without interference cancellation techniques to decode packets simultaneously from different APs. For the case that the packets from different APs do not end at the same time, ACK management from the client device is challenging. Wireless full duplex allows the client device to send an ACK to an AP while still receiving signals from other APs.

\section{A. Interference Nulling}

When multiple APs transmit different packets to a client device, the packets from different APs interfere with one another. However, leveraging the multiple antennas on the client device, we can properly remove the interference and decode each of the intended packets. Taking the system shown in Figure 2 as an example, AP 1 and AP 2 transmit two different packets $x_{1}$ and $x_{2}$, respectively, to the client device. The client device is equipped with 2 antennas. Let $y_{1}$ and $y_{2}$ denote the packets received by the two antennas on the client device, respectively. Then we have:

$$
\begin{aligned}
& y_{1}=h_{11} \times x_{1}+h_{12} \times x_{2}+n_{1} \\
& y_{2}=h_{21} \times x_{1}+h_{22} \times x_{2}+n_{2}
\end{aligned}
$$

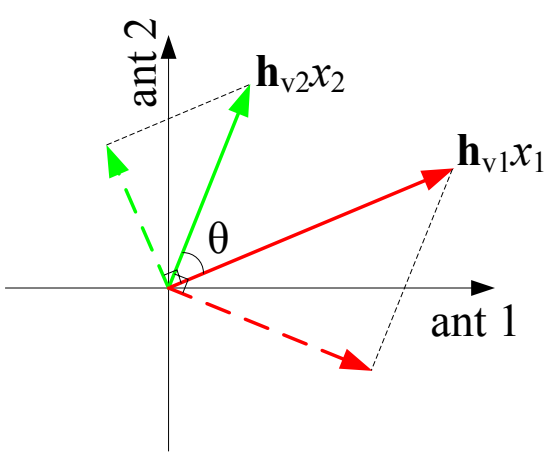

Fig. 3: The channel vectors shown in the plane spanned by the two antennas on the client device.

We can also denote the dual packet reception in matrix form as:

$$
\boldsymbol{Y}=\boldsymbol{H} \boldsymbol{X}+\boldsymbol{N}
$$

where,

$$
\begin{aligned}
\boldsymbol{Y} & =\left[y_{1}, y_{2}\right]^{T} \\
\boldsymbol{X} & =\left[x_{1}, x_{2}\right]^{T} \\
\boldsymbol{H} & =\left[\left[h_{11}, h_{12}\right]^{T},\left[h_{21}, h_{22}\right]^{T}\right]^{T}
\end{aligned}
$$

We can remove the interference and obtain $x_{1}$ and $x_{2}$ by multiplying $H^{-1}$ on both sides of Equation (5) to obtain:

$$
\boldsymbol{X}=\boldsymbol{H}^{-1} \boldsymbol{Y}+\boldsymbol{H}^{-1} \boldsymbol{N}
$$

This could also be explained as channel vector operation used in [8]. We plot the channel vectors $\boldsymbol{h}_{v 1}=\left[h_{11}, h_{21}\right]$ and $\boldsymbol{h}_{v 2}=\left[h_{12}, h_{22}\right]$ on the plane spanned by the two antennas on the client device, as shown in Figure 3. In order to decode $x_{1}$, we project $\boldsymbol{h}_{v 1} x_{1}$ in the direction that is orthogonal to $\boldsymbol{h}_{v 2}$. This nullifies the interference from $x_{2}$, and we can decode $x_{1}$. Similarly, we can we project $\boldsymbol{h}_{v 2} x_{2}$ in the direction that is orthogonal to $\boldsymbol{h}_{v 1}$. This nullifies the interference from $x_{1}$, and we can decode $x_{2}$. Note that because of the projection operation, as discussed in [8], the resulted effective SNR is reduced to $\sin ^{2}(\theta) \mathrm{SNR}_{\text {orig }}$, which is also shown in Figure 3. Here, $\theta$ is the angle between $\boldsymbol{h}_{v 1}$ and $\boldsymbol{h}_{v 2}$ and can be calculated as:

$$
\cos \theta=\frac{\left|\boldsymbol{h}_{v 1} \boldsymbol{h}_{v 2}^{*}\right|}{\left\|\boldsymbol{h}_{v 1}|| \mid \boldsymbol{h}_{v 2}\right\|}
$$

If $\boldsymbol{h}_{v 1}$ and $\boldsymbol{h}_{v 2}$ are orthogonal, $\theta$ will be $\pi / 2$. As discussed in Section III, we have selected the semi-orthogonal APs. Hence, the effective SNR will not significantly degrade.

\section{B. Interference Cancellation}

As discussed above, interference nulling usually results in reduced effective SNR. Another option is to decode the packets from one of the APs by interference nulling. Then, we can reconstruct the transmission and cancel it from the mixed reception to obtain the original packet from another AP. The whole process can be divided into the following steps:

1) Use interference nulling to decode the packet from one of the APs (taking $x_{1}$, for example) and estimate the 
sampling frequency offset, carrier frequency offset, and channel impulse response during the decoding.

2) Encode and modulate the decoded packet and reconstruct the received packet according to the channel response, sampling frequency offset, and carrier frequency offset.

3) Remove the reconstructed packet ( $x_{1}$ for example) from the mixed reception and then decode the remaining packet $\left(x_{2}\right)$ with no interference.

Ideally, using interference cancellation, we can remove the interference of parallel transmissions to obtain the second packet and achieve better performance than simply nulling the interference from the first AP. However, in practice, it is hard to reconstruct the interfering packet without errors, because of the residual carrier frequency error, residual sampling frequency error, and residual channel estimate errors. The reconstruction errors will be the residual interference. As discussed in [6]-[8], the residual interference is usually greater than the noise level. Thus, using interference cancellation, the resulted effective SNR is less than half of the original SNR. Noting that if we use interference nulling on both packets, due to the channel orthogonality of the selected APs, the resulted SNR usually degrades less than 50 percent (i.e., the resulting effective SNR is not as degraded with interference nulling as opposed to interference cancellation). Additionally, the reconstruction of the packets is far more complex and requires far greater computational/hardware resources than the interference nulling. Furthermore, this will also cause substantial delays because of the decoding and reconstruction process. As a result, we only use interference nulling on simultaneous transmissions in the iBeam system, which could significantly reduce the computational complexity while maintaining high throughput.

\section{Compatibility With IEEE 802.11 Standard}

In a WiFi access network, an ACK packet is required when the receiver successfully decodes a unicast data packet. Otherwise, the transmitter will treat the data packet as a transmission failure and re-transmit the same packet. For a corporate network, the APs could be cooperative, either being controlled by a network server or through exchanging control information between one another. In this case, the APs selected by the client device may not necessarily start their transmissions at the same time, but the transmitted packets can be synchronized to end at the same time. Then, the client device can send ACKs to all APs simultaneously. We call this synchronized ACKs.

However, for distributed APs that are not able to be synchronized, their transmissions probabilistically do not end at at the same time. After successfully decoding a packet from one of the APs, the mobile client is required to send an ACK back to that AP. However, for half-duplex wireless devices, receiving the packets from other APs that end later would prevent the ACK packets from being sent back to the AP that had the packet end first. Full duplex wireless communication is able to solve this ACKs problem. With full duplex, the client devices can actually transmit ACK packets while receiving other packets from APs. We call this asynchronized ACKs.
Wireless full duplex has been well studied and practical experiments has shown that it is ready to be used in wireless communications [18], [19].

\section{IMPLEMENTATION AND EXPERIMENTS}

In this section, we evaluate the iBeam system, including the feasibility of our channel reciprocity assumption and the uplink and downlink portions of the iBeam system. The analysis is performed on WARP [12], a software defined radio platform with extensive over-the-air experimentation.

\section{A. Experiment Setup}

We implement and evaluate iBeam on WARPLab, a software defined radio platform which enables users to implement the physical layer and MAC layer functions in MATLAB in the form of software code. Then, the coded and modulated data samples are transferred to the WARP board via an Ethernet cable. WARP is then triggered to transmit data samples over the air. The receiver samples the received signal over the air and then transfers the raw samples to the PC, where the receiver also leverages MATLAB to demodulate and decode the sampled signal to obtain the packets that the transmitter sends. We use one WARP board as the client device, which is equipped with two antennas. To show the practical application of our system, we choose antennas with small size and mount them at approximately $10 \mathrm{~cm}$ apart on a plastic board. The spacing emulates an area of a typical commercial smartphone, as shown in Figure 4. We implemented an OFDM scheme with 64 subcarriers, similar to IEEE 802.11 devices. We use a $10 \mathrm{MHz}$ bandwidth and run the experiments at $2484 \mathrm{MHz}$ carrier frequency. In our system, we can choose between the same 8 bit rates as present in IEEE $802.11 \mathrm{a} / \mathrm{g}$ by setting the code rates and modulation orders, accordingly. For uplink, the client device chooses different packet sizes for different bit rates to synchronize different beams. For downlink, because WARP system does not support wireless full duplex, we use synchronized ACKs in our implementation and evaluation. We control the packets transmission from different APs to start and end at the same time. With the same frame slot, we transmit $\{960,1440,1920,2880,3840,5760,7680,8640\}$ bits for each bit rate, respectively.

Rate adaptation significantly improves the spectrum efficiency in IEEE $802.11 \mathrm{a} / \mathrm{g} / \mathrm{n}$ systems and has been well studied [20], [21]. A MU-beamforming system also requires rate adaptation strategies and algorithms to achieve greater levels of spectrum efficiency. For multi-user systems, rate adaptation must be jointly considered with power allocation and channel variations, which is more challenging than in single-user systems.

For MU-Beamforming, power allocation has multiple rules to follow. For example, an aggressive metric is to maximize the total throughput. Some other metrics could be throughput fairness or transmission time fairness between APs. However, when the user connects to multiple APs via iBeam, the fairness between APs is not as critical from the client's point of view. Thus, the maximum total throughput that the client experiences could be achieved according to the water-filling algorithm. 


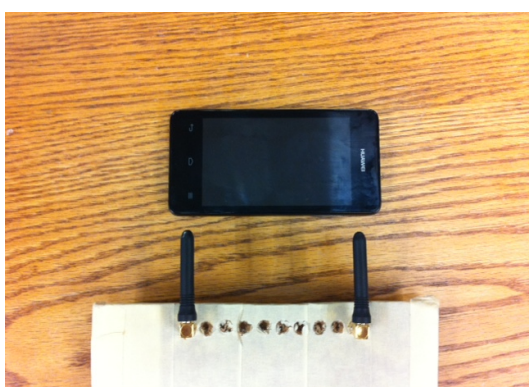

Fig. 4: Antennas used on the client device.

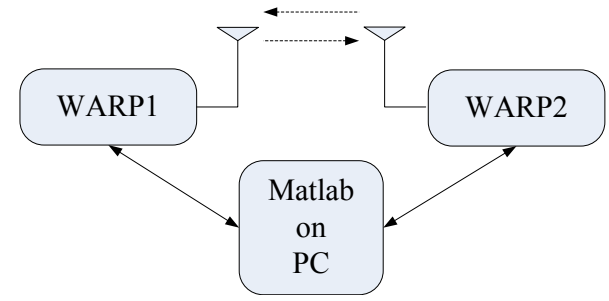

Fig. 5: Channel reciprocity test architecture.

For iBeam, the received signal strength (RSS) depends on both the channel variations and the power allocation. The client device can predict the received signal strength after getting the steering matrix and the power allocation. As a result, the RSS-based rate adaptation is the most straightforward method for uplink (e.g., the one we used in [22]). According to the received signal strength, an optimal bit rate can be selected to both ensure the packet delivery and maximize the spectrum efficiency. For downlink, the APs can use packetloss-based rate adaptation algorithm, which is the predominant rate adaptation algorithm implemented in commercial APs.

\section{B. Channel Reciprocity Evaluation}

For client-side beamforming to one or more APs, uplink channel state information is required by the client device. Further, interference nulling requires downlink channel state information. For protocols with CSI feedback defined (e.g. IEEE 802.11n, IEEE 802.11ac), both implicit CSI feedback and explicit CSI feedback can be used to get the estimated channel parameters and construct the steering matrix for the beamformer. In implicit feedback mode, the beamformer relies on channel reciprocity in the time division duplex channel to estimate the channel from the beamformer to the beamformee. In iBeam, we leverage the downlink channel estimation to calculate the uplink channel state information. This is necessary for networks without CSI feedback and could also avoid the repeated CSI feedback for networks with CSI feedback, improving the system efficiency. Note that channel reciprocity only applies in wireless channels. For the channel between the transmitter and the receiver, however, the digital and analog components through which the signal passes could also be regarded as part of the channel, which makes the overall channel no longer reciprocal. Sufficient levels of training is needed to calibrate and compensate for the the amplitude and phase offset, and to recover the reciprocity of the overall channel. A channel reciprocity calibration has been proposed

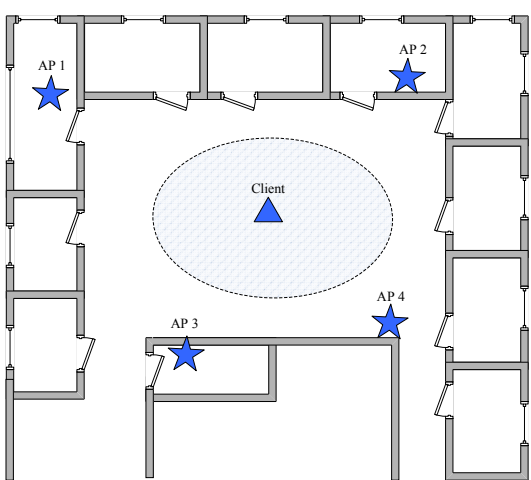

Fig. 6: Floor plan of the nodes in the experiment.

in [23] and experimentally evaluated in [24], which shows an acceptable accuracy level. However, the calibration accuracy highly depends on the accuracy of the channel estimates during the calibration process, which in turn, depends on the channel quality. Let $\hat{H}_{A B}$ and $\hat{H}_{B A}$ denote the channel estimate from station A to station $\mathrm{B}$ and the channel estimate from station $\mathrm{B}$ to station A during the calibration, respectively. Assuming $\tilde{H}_{A B}$ is the instantaneous channel estimate from station A to station $\mathrm{B}$, we calculate $\tilde{H}_{B A}$, the channel estimate from station $\mathrm{B}$ to station A, as:

$$
\begin{gathered}
\tilde{H}_{B A}=\left(\tilde{H}_{A B}+E_{A B}\right) K_{A B} \\
K_{A B}=\frac{\hat{H}_{B A}+E_{B A}}{\hat{H}_{A B}+E_{A B}}
\end{gathered}
$$

Here, $K_{A B}$ is the calibration parameter to calculate $\tilde{H}_{B A}$ from $\tilde{H}_{A B}$, and $E_{A B}, E_{B A}$ are the channel estimation errors due to the additive Gaussian noise in the system.

The residual calibration errors affect each packet transmission. When the channel quality is poor during calibration, the result would be with more errors, resulting in poor performance for the following packet transmission. A simple way for calibration with higher accuracy is to perform the calibration only when the received SNR is higher than a threshold. Each client device needs to be precalibrated by an AP with CSI feedback, this could be done during manufacturing or by the user during daily use.

We use two WARP boards sending signals back and forth to evaluate the performance and accuracy of the CSI estimation based on channel reciprocity. The PC connected to both WARP boards controls one board as the transmitter and the other as a receiver. We then alternate the sender and receiver every other transmission, as shown in Figure 5. We change the transmit power to see how the received signal strength affects the calibration accuracy. For each transmit power settings, we repeat the experiment 500 times for the calibration parameter calculation and obtain the normalized variance of the 500 trials. We show the calibration variances versus the received SNR in Figure 7a. We can see that, with low SNR, the $K$ parameter has large variances, leading to large channel estimation errors via channel reciprocity. For the case when the user is connected to two APs, we simulate the $K$ parameter variance versus the MU-Beamforming capacity efficiency, as 


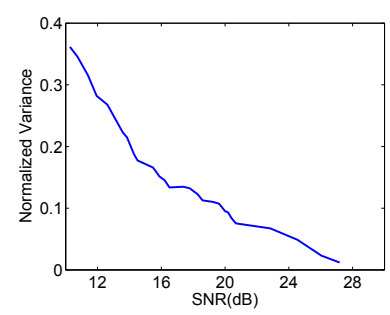

(a) Channel reciprocity calibration errors versus channel quality.

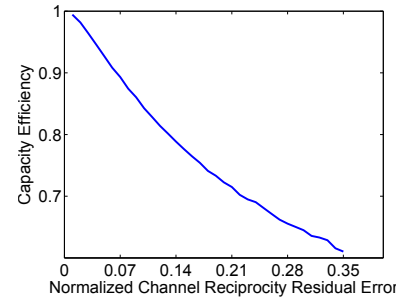

(b) Capacity efficiency versus channel reciprocity calibration errors.
Fig. 7: Performance evaluation of channel-reciprocity-based CSI acquisition.

shown in Figure $7 \mathrm{~b}$. With a normalized variance of 0.1 for $K$, corresponding to link quality (SNR) of at least $16 \mathrm{~dB}$, the capacity of the MU-beamforming system will degrade approximately 10 percent.

\section{Uplink Throughput Evaluation}

For the uplink direction, the client device transmits multiple streams to multiple APs simultaneously by MU-beamforming. We use four WARP boards as the APs, and each AP operates with one antenna. Another WARP board is equipped with two antennas and acts as the client device. We place both the APs and the client device in an indoor laboratory, as shown in Figure 6. The stars indicate the locations of the APs, which do not change during the experiments. The triangle indicates the client device, which randomly selects locations within the gray, oval-shaped region. We select 20 locations for the client device. For each location, we test the throughput of three schemes. In the first scheme, we force the client device to use one antenna to connect to the AP with the best channel gain, which we call SISO. In the second scheme, we force the client device connect to the AP with the best channel gain with single-user beamforming, which we call SU-Beamforming. In the third scheme, we force the client device connect to multiple APs with MU-beamforming. For all three schemes, we use the same total transmit power from the client device. The throughput for different schemes are shown in Figure 8a and Figure $8 b$.

We can see that, with only two antennas equipped on the client device, we can achieve significant throughput improvement. With the same total transmit power, the MUbeamforming transmission outperforms the SU-beamforming mode more than 30 percent in terms of throughput. The MUbeamforming mode outperforms the SISO mode more than 50 percent in terms of throughput. Nonetheless, there are cases in Figure $8 \mathrm{a}$ and $8 \mathrm{~b}$ where the throughput of MU-beamforming is less than SISO or SU-beamforming due to the channel vectors of the 2 APs being less orthogonal. Here, in order to satisfy the zero-forcing requirement, the resulted beams could not directly point to the intended AP, leading to a low received signal strength at both APs. However, for SU-beamforming, the signals from different transmit antennas can always be constructively added together by properly aligning the carrier phase on the transmit antennas. Thus, in these cases, SUbeamforming outperforms MU-beamforming.

\section{Downlink Throughput Evaluation}

We use the same system settings and methods as in the uplink setting to evaluate and compare the downlink performance. In this case, we compare the throughput of two schemes. The first scheme is to force the AP with the best channel gain to transmit the data packets to the client device. The second scheme allows two APs to transmit different packets simultaneously to the client device. For each AP, the transmit power remains the same for the two schemes, meaning that the 2-AP scheme has a total transmit power of twice as much as the signal AP scheme. From the clients point of view (which is usually powered by a battery), the same power is consumed for reception. From the AP's point of view (which is usually hard-wired to a power source), this transmit power difference is negligible. We randomly select 30 locations for the client device. At each location, we measure the throughput of iBeam and single-AP connection. Then, we plot the throughput in Figure 8c. We can see that, with two-AP transmission, the downlink throughput is improved as much as 80 percent. However, similar to the uplink direction, we can see cases where the throughput with multiple APs connected is less than the SISO scheme. This also results from the non-orthogonality of the channels from different APs to the client device. As we discussed in Section IV, the signal projection causes signal strength degradation from each AP. If the channel gain of second AP is far worse than the first AP, the capacity gain from the second AP may not compensate for the projection loss of the first AP, leading to a lower throughput than the SISO scheme.

\section{E. Handoff Evaluation}

When the client device moves, the channel gains to each AP change with time. For a single-AP system, when the client device moves out of the range of the current connected AP, handoff occurs. However, the client is disconnected until the client device is associated with another AP. This handoff process usually takes as long as hundreds of milliseconds to even several seconds, depending on the handoff algorithms, implementation, and traffic load of the network [17]. The frequency of handoff depends on the speed at which the client device travels. The faster the client devices moves, the more frequently handoffs occur, leading to an increasingly unstable connection. In our design, the client device is connected to two APs at the same time. When there is a handoff for one of the APs, the other AP is still in service to avoid the outage typically experienced with mobile handoff.

The traditional handoff process includes channel scanning, authentication, and re-association as well as any updates necessary on the wired network. Channel scanning consumes most of the handoff time because there are potentially tens of channels to scan. The client devices send probe packets, listen to feedback packets, and switch to another channel. Authentication, re-association, and wired network updates take less than $40 \mathrm{~ms}$ in total [17]. For the iBeam system, both the client devices and the APs work on the same channel. As a 


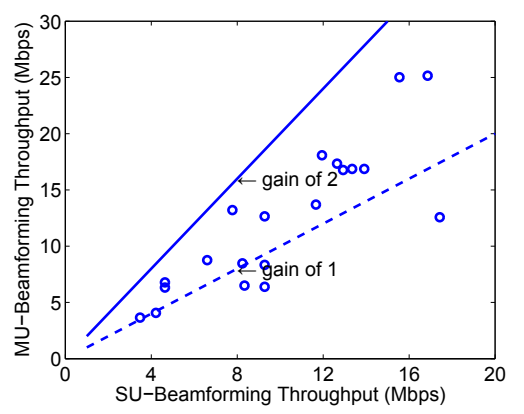

(a) Throughput comparison between SUbeamforming and MU-beamforming.

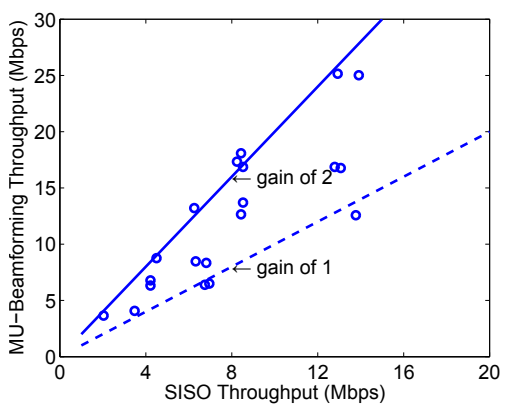

(b) Throughput comparison between SISO mode and MU-beamforming.

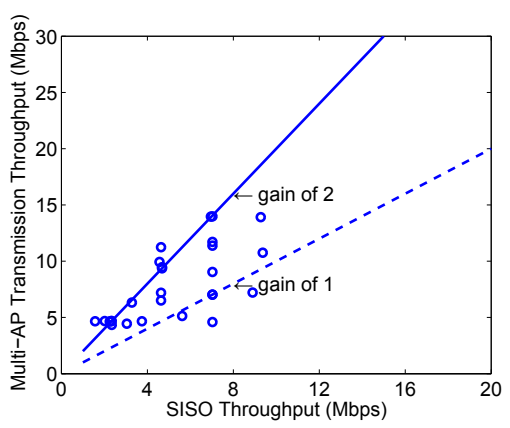

(c) Throughput comparison between SISO mode and multi-AP transmission.

Fig. 8: Throughput evaluation of iBeam versus traditional multi-AP schemes.

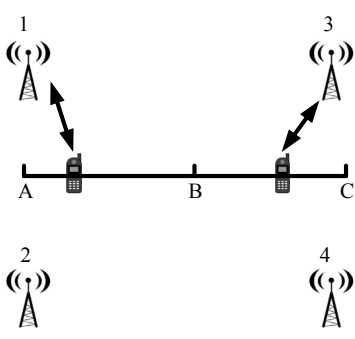

(a) TD scheme model.

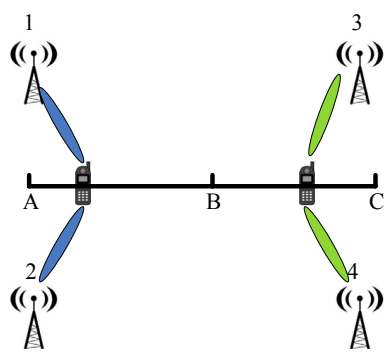

(b) iBeam model.
Fig. 9: AP handoff model.

result, the probing delay is significantly reduced. The total handoff delay for iBeam is less than $60 \mathrm{~ms}$ (assuming the maximum probe delay is $20 \mathrm{~ms}$, according to [17]).

Although WARPLab transmits signals over the air, it cannot provide real-time interaction with commercial APs due to different inter-frame spacing and insufficient turn-around time. Thus, we create a simple network topology to demonstrate the handoff performance of iBeam versus time-division (TD) multi-AP systems. As shown above, with only 2 antennas on the client device, we obtain about 1.5 times uplink throughput gain and about 1.8 times downlink throughput gain. Assuming that the uplink and downlink offered load are equal, then the average total throughput gain of iBeam is about 1.65 times of the time-division scheme. In our model, we assume the average throughput of the time-division scheme is normalized to 1. Then, the iBeam throughput is normalized to 1.65.

As shown in Figure 9a, the client device moves from location $\mathrm{A}$ to $\mathrm{C}$. For the time-division scheme, it connects to AP 1 when it moves from location A to $\mathrm{B}$, and connects to AP 3 when it moves from location $B$ to $C$. The handoff occurs when the client device is crossing location B. In Figure 9b, for iBeam, the client device connects to AP 1 and AP 2 when it moves from location $\mathrm{A}$ to $\mathrm{B}$, and transfers to AP 3 and AP 4 when it moves from location B to C. Note that, for iBeam, the client device first makes handoff between AP 2 and AP 4. After being associated to AP 4, the client device then hands off between AP 1 and AP 3. When the client device is at location A, the received signal strength is $S_{A}$.

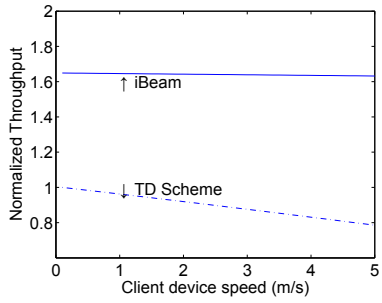

(a) Throughput affected by handoff.

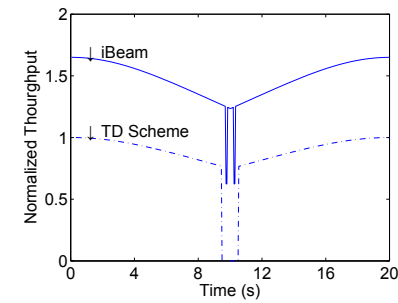

(b) Outage caused by handoff.
Fig. 10: Handoff performance comparison between iBeam and TD scheme.

Assuming that location A is the midpoint of AP 1 and AP 2 and location $\mathrm{C}$ is the midpoint of AP 3 and AP 4, when the client moves a distance $x$ towards $\mathrm{C}$, the received signal strength is then $S_{x}=S_{A} d^{2} /\left(4 x^{2}+d^{2}\right)$, with $d$ denoting the distance between AP 1 and AP 2. Then, the instantaneous capacity is $B \log _{2}\left(1+S_{x} / N\right)$. We can calculate how the handoff affects the system throughput.

In our simulation, we set $d=20 \mathrm{~m}$ and we assume the time-division scheme has a handoff time of one second. We calculate the average throughput of both schemes when the client device moves from location $\mathrm{A}$ to $\mathrm{C}$, with different speed. We also simulate the instantaneous throughput of both schemes when the client devices moves at a speed of $1 \mathrm{~m} / \mathrm{s}$. As shown in Figure 10a and 10b, we simulate how handoff affects the communication for the two schemes. We can see that, because of the small handoff time, the iBeam throughput is largely independent of client speed. However, the throughput of the time-division scheme drops significantly when the speed increases since the portion of outage time is correspondingly increased. Moreover, for iBeam, because there is at least one AP connection at any time, there is no outage during the handoff. However, for the time-division scheme, there is outage for each handoff.

\section{RELATED WORK}

The majority of the work on multi-user communication has been from the network to the client device. This includes coordinated multipoint to point or network MIMO techniques 
in cellular networks [25], as well as wireless LANs [7], [13], [24]. These schemes require a high-speed central controller that is connected to each AP and antennas in a cellular or enterprise wireless network, which does not exist in mesh deployments or when WiFi APs belong to different service providers. Other work has focused on enhancing the performance of mobile devices through communication with multiple APs that belong to different access technologies [26]-[28], or the same technology but tuned to non-overlapping frequency channels [9], [29]-[31]. Other work has proposed methods that use directional antennas or beamforming techniques at the mobile device [3], [32], [33], but these works are limited to single-AP communication. In contrast, we design and implement a system that leverages the increasing computational and communication resources of client-side devices to provide the high capacity and reliability gains associated with multi-user communication to multiple access points while consuming a small spectral footprint.

\section{CONCLUSION}

In this paper, we designed iBeam, an intelligent clientside MIMO framework to leverage simultaneous use of multiple access points. For the uplink traffic, we leveraged MUbeamforming technology to enable the client devices to transmit multiple data streams to multiple APs simultaneously. For downlink, we used interference nulling to enable the client devices to decode parallel transmissions from multiple APs. With MIMO techniques applied to both uplink and downlink directions, we significantly improved the system throughput due to the multi-user and beamforming gains. With only two antennas on the client device with antenna spacing comparable to a smartphone, iBeam achieves an average improvement of 50 percent for the uplink traffic and 80 percent for downlink traffic. This improvement will only increase with the use of more antennas or greater antenna separation, which could be offered by non-mobile clients. For mobile users, the stability and reliability are significantly improved due the multi-user diversity across APs.

\section{ACKNOWLEDGEMENTS}

We would like to thank the anonymous reviewers for their valuable feedback, which improved the paper. This work was supported in part by NSF grants: CNS-1150215, CNS1318607, and CNS-1320442.

\section{REFERENCES}

[1] E. Aryafar, N. Anand, T. Salonidis, and E. W. Knightly, "Design and experimental evaluation of multi-user beamforming in wireless lans," in Proceedings of MobiCom, 2010.

[2] IEEE Std 802.11ac, in process and expected in 2014.

[3] H. Yu, L. Zhong, A. Sabharwal, and D. Kao, "Beamforming on mobile devices: a first study," in Proceedings of MobiCom, 2011.

[4] E. Aryafar, M. Khojastepour, K. Sundaresan, S. Rangarajan, and E. Knightly, "Adam: An adaptive beamforming system for multicasting in wireless lans," in Proceedings of INFOCOM, 2012.

[5] T. Yoo and A. Goldsmith, "On the optimality of multiantenna broadcast scheduling using zero-forcing beamforming," Selected Areas in Communications, IEEE Journal on, vol. 24, no. 3, pp. 528-541, March 2006.

[6] K. C.-J. Lin, S. Gollakota, and D. Katabi, "Random access heterogeneous mimo networks," in Proceedings of SIGCOMM, 2011.
[7] K. Tan, H. Liu, J. Fang, W. Wang, J. Zhang, M. Chen, and G. M. Voelker, "Sam: enabling practical spatial multiple access in wireless lan," in Proceedings of MobiCom, 2009.

[8] W.-L. Shen, Y.-C. Tung, K.-C. Lee, K. C.-J. Lin, S. Gollakota, D. Katabi, and M.-S. Chen, "Rate adaptation for 802.11 multiuser mimo networks," in Proceedings of Mobicom, 2012.

[9] S. Kandula, K. C.-J. Lin, T. Badirkhanli, and D. Katabi, "Fatvap: aggregating ap backhaul capacity to maximize throughput," in Proceedings of NSDI, 2008.

[10] A. Balasubramanian, R. Mahajan, A. Venkataramani, B. N. Levine, and J. Zahorjan, "Interactive wifi connectivity for moving vehicles," SIGCOMM Comput. Commun. Rev., vol. 38, no. 4, Aug. 2008

[11] A. Adya, P. Bahl, J. Padhye, A. Wolman, and L. Zhou, "A multi-radio unification protocol for ieee 802.11 wireless networks," in Proceedings of Broadband Networks, 2004.

[12] "Rice university wapr project." [Online]. Available: http://warp.rice.edu

[13] H. S. Rahul, S. Kumar, and D. Katabi, "Jmb: scaling wireless capacity with user demands," in Proceedings of SIGCOMM, 2012.

[14] R. Chandra, P. Bahl, and P. Bahl, "Multinet: connecting to multiple ieee 802.11 networks using a single wireless card," in INFOCOM 2004. Twenty-third AnnualJoint Conference of the IEEE Computer and Communications Societies, 2004.

[15] G. Caire and S. Shamai, "On the achievable throughput of a multiantenna gaussian broadcast channel," Information Theory, IEEE Transactions on, vol. 49, no. 7, pp. 1691-1706, 2003.

[16] H. Viswanathan, S. Venkatesan, and H. Huang, "Downlink capacity evaluation of cellular networks with known-interference cancellation," IEEE J.Sel. A. Commun., vol. 21, no. 5, pp. 802-811, Sep. 2006

[17] I. Ramani and S. Savage, "Syncscan: practical fast handoff for 802.11 infrastructure networks," in Proceedings of INFOCOM, 2005.

[18] D. Bharadia, E. McMilin, and S. Katti, "Full duplex radios," in Proceedings of SIGCOMM, 2013.

[19] E. Aryafar, M. A. Khojastepour, K. Sundaresan, S. Rangarajan, and M. Chiang, "Midu: enabling mimo full duplex," in Proceedings of Mobicom, 2012.

[20] M. Lacage, M. Hossein, and T. Turletti, "IEEE 802.11 rate adaptation: A practical approach," Master's thesis, October 2004.

[21] D. Halperin, W. Hu, A. Sheth, and D. Wetherall, "Predictable 802.11 packet delivery from wireless channel measurements," in Proceedings of SIGCOMM, 2010.

[22] J. Camp and E. Knightly, "Modulation rate adaptation in urban and vehicular environments: Cross-layer implementation and experimental evaluation," Networking, IEEE/ACM Transactions on, vol. 18, no. 6, pp. $1949-1962$, dec. 2010.

[23] "System description and operating principles for high throughput enhancements to 802.11," in IEEE 802.11-04/0870r, 2004.

[24] S. Gollakota, S. D. Perli, and D. Katabi, "Interference alignment and cancellation," in Proceedings of SIGCOMM, 2009.

[25] J. Lee, Y. Kim, H. Lee, B. L. Ng, D. Mazzarese, J. Liu, W. Xiao, and Y. Zhou, "Coordinated multipoint transmission and reception in lteadvanced systems," Communications Magazine, IEEE, 2012.

[26] S. Deb, K. Nagaraj, and V. Srinivasan, "Mota: engineering an operator agnostic mobile service," in Proceedings of MobiCom, 2011.

[27] P. Coucheney, C. Touati, and B. Gaujal, "Fair and efficient usernetwork association algorithm for multi-technology wireless networks," in Proceedings of INFOCOM, 2009.

[28] W. Wang, X. Liu, J. Vicente, and P. Mohapatra, "Integration gain of heterogeneous wifi/wimax networks," Mobile Computing, IEEE Transactions on, 2011

[29] B. Kauffmann, F. Baccelli, A. Chaintreau, V. Mhatre, K. Papagiannaki, and C. Diot, "Measurement-based self organization of interfering 802.11 wireless access networks," in Proceedings of INFOCOM, 2007.

[30] L. Li, M. Pal, and Y. Yang, "Proportional fairness in multi-rate wireless lans," in Proceedings of INFOCOM, 2008

[31] H. Soroush, P. Gilbert, N. Banerjee, M. D. Corner, B. N. Levine, and L. Cox, "Spider: improving mobile networking with concurrent wi-fi connections," in Proceedings of SIGCOMM, 2011.

[32] A. Amiri Sani, L. Zhong, and A. Sabharwal, "Directional antenna diversity for mobile devices: characterizations and solutions," in Proceedings of MobiCom, 2010.

[33] V. Navda, A. P. Subramanian, K. Dhanasekaran, A. Timm-Giel, and S. Das, "Mobisteer: using steerable beam directional antenna for vehicular network access," in Proceedings of MobiSys, 2007. 